\title{
Grouping in the shine-through effect
}

\author{
Michael H. Herzog \\ École Polytechnique Fédérale de Lausanne (EPFL), Lausanne, Switzerland \\ AND \\ Ulrike Schmonsees, Jakob M. Boesenberg, Timm Mertins, \\ AND MANFRED FAHLE \\ Human Neurobiology, Bremen, Germany
}

\begin{abstract}
How the elements of a visual scene are grouped into objects is one of the most fundamental but still poorly understood questions in visual neuroscience. Most investigations of perceptual grouping focus on static stimuli, neglecting temporal aspects. Using a masking paradigm, we show that the neural mechanisms underlying grouping seem to be both fast and complex. For example, a vernier target was followed by, first, a briefly presented grating and, then, a long-lasting, extended grating. Under these conditions, the briefly presented grating is hardly visible. Still, vernier discrimination strongly changed with the number of elements of the briefly displayed grating being worst for small gratings. In accordance with a neural network model of masking, we propose that the edges of the briefly presented grating and the vernier interfere in spite of the short presentation time. We suggest that this fast edge processing is a first step for unconscious grouping processes.
\end{abstract}

How the human brain distills objects from a visual scene is an unresolved problem. For example, a treetop may contain thousands of single leaves. In spite of each leaf's being an object by itself, the treetop is perceived as a coherent object and not just as a collection of single leaves. Grating stimuli are another example. Although a grating is composed of single bars, separated from each other, the observer perceives one object segmented from its background even though the bars are not connected with each other. Whereas the spatial aspects of this perceptual grouping are well investigated (for a review, see Palmer, Brooks, \& Nelson, 2003), its temporal aspects have been studied to a much lesser degree (e.g., Caputo, 1996; Kurylo, 1997).

Here, we investigate the dynamics of spatiotemporal interactions that may underlie the grouping of single lines into a grating. For this purpose, we use a version of a backward masking effect that we call shine-through (Herzog \& Koch, 2001). A single vernier shines through a following grating if the grating comprises more than seven aligned verniers (Figure 1). For smaller gratings or for extended inhomogeneous gratings, no shine-through occurs, and discrimination of the vernier offset strongly deteriorates (Figure 1; Herzog \& Koch, 2001). For example, inserting two gaps into a grating of 25 elements dramatically raises thresholds (Figure 1).

In accordance with physiological and psychophysical studies (MacKay, 1973; Macknik, Martinez-Conde, \& Haglund, 2000; McCarter \& Roehrs, 1976; Sagi \& Hochstein, 1985), we hypothesized that the neural activity in- volved in representing edges is stronger than the neural activity for representations of homogeneous, repetitive stimulus parts such as the inner elements of gratings. Computer simulations showed indeed that an enhanced activity corresponding to the representation of edges can dynamically suppress the activity corresponding to that for the vernier when an edge is close to the vernier (Figure 1, lowest row; Hermens, Luksys, Gerstner, Herzog, \& Ernst, 2008; Herzog, Ernst, Etzold, \& Eurich, 2003; see also Zhaoping, 2003). For extended homogeneous gratings, shine-through occurs, because the edges are remote and the inner grating elements are suppressed.

We suggest that these computational processes underlie a first step of grouping. For example, observers do not perceive the "gap grating" as one grating containing gaps, but rather as three smaller gratings. In the computer simulations, the edges of all three gratings are enhanced while the inner grating elements are suppressed. In this sense, vernier suppression is a side effect of the grouping processing, depending on whether or not an "irregularity" is close to the vernier.

To understand the dynamics of these grouping processes better, we presented a vernier either followed or preceded by a briefly displayed grating. Both of these sequences were followed by the long-lasting standard grating of Figure 1A. First, we tested the hypothesis that edge computation is fast. If this hypothesis is true, one would expect varying effects, depending on the number of elements when the briefly displayed grating follows the vernier (Figure 2). Strongest suppression would be

M. H. Herzog, michael.herzog@epfl.ch 


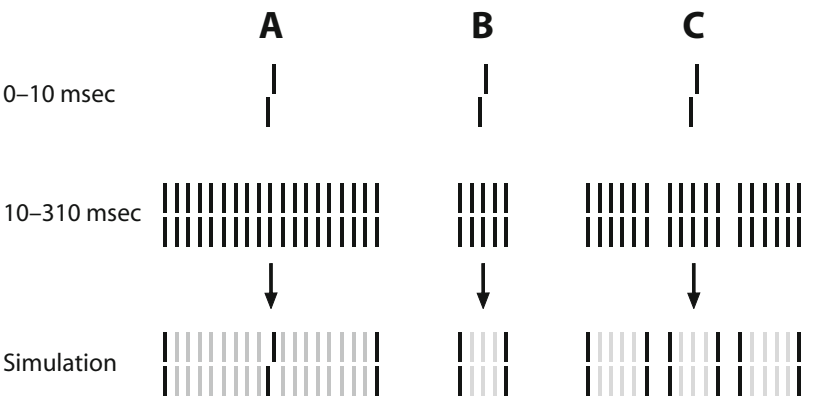

Figure 1. A vernier is followed by various kinds of gratings lasting for 300 msec. (A) If a grating contains more than seven elements, the vernier shines through the grating. For gratings with less than seven elements (B) or gratings containing gaps $(C)$, no shine-through occurs, and vernier discrimination strongly deteriorates. On a descriptive level of perceptual organization, we propose that grouping may explain these effects. For example, the gaps segment the entire array into smaller gratings (grouping by proximity). Hence, three smaller gratings are perceived instead of one larger grating with gaps. On a neural description level, we propose that neural activity corresponding to the representation of the inner elements of the gratings is dynamically suppressed (light gray elements in the row "Simulation"), whereas the activity corresponding to grating edges or inhomogeneities, such as gaps, is strongly enhanced (black elements; Hermens, Luksys, Gerstner, Herzog, \& Ernst, 2008; Herzog, Ernst, Etzold, $\&$ Eurich, 2003). Neural activity corresponding to representations of the vernier is reduced when edges or gaps are close to the vernier. With the 25-element grating, shine-through occurs because edges are remote and the neural activity for the inner elements of the gratings is suppressed.

expected for small gratings, because the edges are closer to the vernier. A second hypothesis states that the dynamic suppression of inner elements of the grating is fast as well. If this is true, one would expect that an extended grating preceding the vernier would yield shine-through, because its inner elements are suppressed before the vernier processing starts. Third, we hypothesized that the presentation of more than one target vernier would yield weaker performance than would one target vernier, because the corresponding vernier activities would mutually inhibit each other. We found evidence for the first and third hypotheses, but not for the second. Our paradigm revealed dynamic effects that are not visible without masking.

\section{GENERAL METHOD}

\section{Participants}

The first four authors and two graduate students of the University of Bremen participated (mean age about 23 years). The students were told that they could quit the experiment at any time they wished. After participants had signed a consent form, visual acuity was determined by means of the Freiburg Visual Acuity Test (Bach, 1996). To participate in the experiments, participants had to reach a value of 1.0 (corresponding to 20/20) for at least one eye.

\section{Apparatus}

Stimuli were displayed on a point-plot device (HP 1334 A) controlled by a Power Macintosh computer via fast 16-bit D/A converters (1-MHz pixel rate). Participants observed the stimuli from a distance of $2 \mathrm{~m}$ in a room illuminated dimly by a background light $(0.5 \mathrm{~lx})$. Luminance of the stimuli was around $80 \mathrm{~cd} / \mathrm{m}^{2}$. Before the stimuli were presented, a fixation dot was turned on in the center of the screen simultaneously with four markers at the corners of the screen for $1 \mathrm{sec}$, followed by a blank screen for $200 \mathrm{msec}$. Refresh time was either 5 or $10 \mathrm{msec}$.

One or more vertical target verniers were presented. Gratings followed or preceded the vernier(s). The grating elements were aligned verniers with all spatial parameters being identical to the those for the target vernier(s) except for the horizontal offset. Segments of verniers were $10^{\prime}$ long separated by a small vertical gap of $60^{\prime \prime}$. Thus, verniers and grating elements were $21^{\prime}$ long. The horizontal spacing between grating elements or target verniers was $200^{\prime \prime}$ in all but one experiment. Single verniers, central verniers of an array of target verniers, and the central element of the gratings appeared in the center of the screen.

If only a single target vernier precedes the grating with 25 elements, the vernier shines through the grating (see Figure 1; Herzog \& Koch, 2001). We call this condition the standard condition, and the grating is called the standard grating. Most of the experiments presented here were variants of this standard condition. For example, we briefly presented an additional grating between the target vernier and the standard grating. In all the experiments, the standard grating lasted for $300 \mathrm{msec}$, whereas the additional gratings were presented for either 10 or $20 \mathrm{msec}$, depending on the observer. verniers and gratings followed or preceded each other immediately; that is, the interstimulus interval was zero.

\section{Procedure}

Observers were asked to discriminate the offset direction (left vs. right) of the vernier(s) shining through the grating by pressing the appropriate one of two push buttons. Incorrect responses were followed by an auditory error signal produced by the computer.

We determined thresholds of $75 \%$ correct responses via the adaptive procedure parameter estimation with sequential testing (PEST; Taylor \& Creelman, 1967). If the vernier offset cannot be identified, PEST offers increasingly large offsets. In order to avoid too large offsets, we restricted the PEST procedure to a maximum offset size of the target vernier of 300 " (that is 1.5 times wider than the horizontal spacing between grating elements). The starting value of PEST 

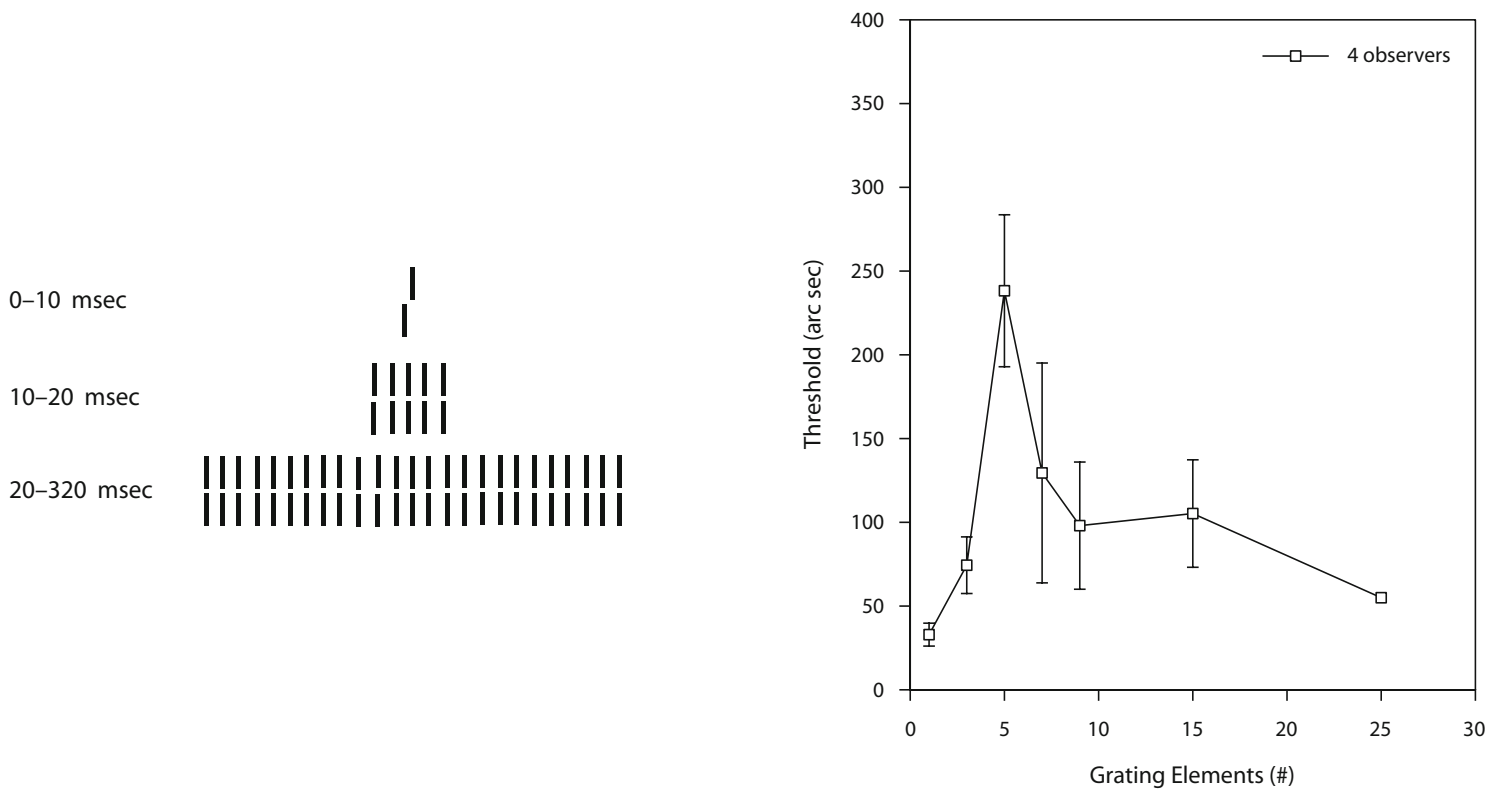

Figure 2. A single vernier preceded a briefly presented grating (10 or $20 \mathrm{msec}$, depending on the observer), which itself was followed immediately by the standard 25-element grating lasting for $300 \mathrm{msec}$. The number of elements of the interleaved grating was varied (for convenience, a single aligned vernier following the target vernier is also called a grating; " 1 " on the abscissa). The left panel shows an example of an interleaved grating with 5 elements. Performance is worst for interleaved gratings with 5 elements and improves for more or fewer elements. Means and standard errors are presented for 4 observers.

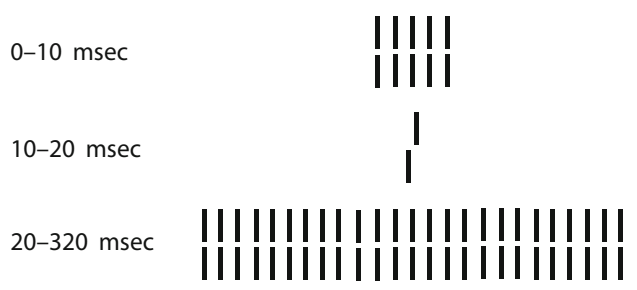

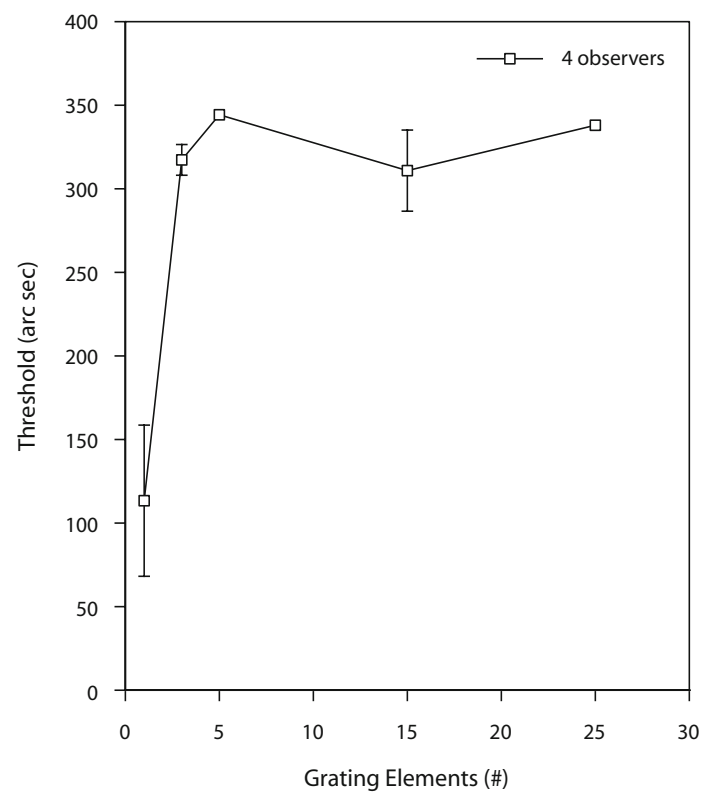

Figure 3. The target vernier was preceded by a grating comprising a varying number of aligned verniers. The vernier was followed immediately by the standard grating. The left panel shows an example of a preceding grating with 5 elements. Shine-through is completely abolished, and performance strongly deteriorates for all preceding gratings except in the one-element condition. Observers were the same as in Figure 2. 
was set to $150^{\prime \prime}$. If observers were unable to reach a threshold value within the predetermined offset range, we assumed invisibility of the vernier target and an offset of $350^{\prime \prime}$ was recorded if the following three criteria were fulfilled. First, PEST had offered offsets increasing in a monotonic fashion. Second, an offset value of $300^{\prime \prime}$ had been provided at least once, and third, the hit rate for the $300^{\prime \prime}$ offset had been below $75 \%$ correct responses. In ambiguous cases, the block of presentation was repeated. For invisible verniers, PEST in general fails to compute a threshold value for obvious reasons. In the rare cases in which PEST computed a value above $350^{\prime \prime}$, this value was still substituted in order to avoid numerical dominance of the value in the averaging process. It should be mentioned that standard errors can be artificially small if in a particular condition the verniers are invisible for all (or some) participants because a value of 350 " was recorded for all of them (see, e.g., Figure 3).

For each participant, every condition was measured twice. Unless stated otherwise, the order of conditions was randomized individually between observers to reduce possible hysteresis or order effects in the averaged data. After every condition had been measured once, the order of conditions was reversed for the second row of measurements in order to compensate at least partly for possible learning effects or fatigue. The two corresponding thresholds were subsequently collapsed.

For every observer, we aimed to find the minimal time (i.e., the shortest duration of the preceding vernier) for which shine-through occurred in the standard condition (Figure 1A). This time was used in all experiments. Presentation time was $20 \mathrm{msec}$ for 3 of the observers and $10 \mathrm{msec}$ for the remaining ones. Each block contained 80 stimulus presentations.

\section{FORWARD VERSUS BACKWARD MASKING}

To test the first two hypotheses - namely the strengthening of outer edges and the weakening of redundant elements during the grouping process - we presented gratings briefly before or after a single vernier (Figures 2 and 3 ).

\section{Method}

Four observers participated in the first experiment. A single target vernier preceded or followed a grating comprising a varying number of aligned verniers ranging from 1 to 25 (for convenience, an aligned vernier is also called a grating). The presentation durations of the target vernier and the grating were identical (for 3 observers, $10 \mathrm{msec}$; for $1 \mathrm{observer}, 20 \mathrm{msec}$ ).

Immediately after the sequence of either target vernier first, followed by the grating, or the grating first, followed by the target vernier, the standard grating was displayed for $300 \mathrm{msec}$ (Figures 2 and 3).

In a control condition, we presented the target vernier either preceded or followed by a 5- or 25-element grating with the same duration as that of the vernier (i.e., the 300 -msec standard grating was not presented). The conditions in which a grating with 5 or 25 elements followed the vernier are denoted with " $\mathrm{v}-5 \mathrm{~m}$ " or "v-25m," respectively. If the gratings preceded the vernier, denotation is " $5 \mathrm{~m}-\mathrm{v}$ " or " $25 \mathrm{~m}-\mathrm{v}$."

Thresholds for the target vernier alone were also determined (v).

\section{Results and Discussion}

When the vernier was followed by the interleaved grating, thresholds varied nonmonotonically, depending on the number of elements with a peak at 5 elements (Figure 2). Even though the interleaved grating is largely invisible, the human brain clearly processes the grating differently, depending on the number of elements. These results are in good accordance with the first hypothesis of a fast and unconscious enhance- ment of neural activity corresponding to the representation of edge elements (see also Herzog, Koch, \& Fahle, 2001a).

When the vernier was preceded by a forward masking grating, performance very strongly deteriorated for 3 or more grating elements. This result strongly disagrees with the second hypothesis stating that at least for the 25 -element preceding grating, thresholds should be only slightly elevated.

The effects of the last two conditions were only visible if the standard grating followed after the target vernier and the briefly presented grating (or vice versa). Without the standard grating, performance was comparable in forward and backward masking and performance strongly improved (Figure 4).

Performance with a grating of 25 elements was very similar to that for the condition with only a single, unmasked vernier (v) while thresholds were elevated for a grating of 5 elements. However, this deterioration of performance was mainly due to 1 participant (see large standard error).

\section{ARRAYS OF TARGET VERNIERS}

\section{Vernier Arrays}

The third hypothesis stated that more target verniers would yield weaker performance than would only one vernier, because the neural activities corresponding to the verniers would interact with each other and thus weaken themselves mutually. This prediction was tested in the second experiment.

\section{Method}

A variable number of offset verniers was presented with a horizontal spacing between verniers of 200". Immediately after the verniers, the standard grating was presented for $300 \mathrm{msec}$ (Figure 5).

Five observers participated. For each observer, his or her minimal presentation time was used $(10 \mathrm{msec}$ for 2 and $20 \mathrm{msec}$ for 3 observers). Observers were free to base their decisions on any cue. Only if 25 verniers preceded the standard grating, observers were asked to look at the center (depending on offset direction, one segment of the outermost verniers was not completely covered by the corresponding standard grating element)

For 3 out of the previous 5 observers plus an additional participant, we presented a varying number of verniers without a grating following. Observers were free to base their decisions on any cue (e.g., on one of the edge elements of the vernier array). verniers were presented with the minimal presentation time that produced shinethrough in the standard condition.

\section{Results and Discussion}

Subjectively, shine-through is clearly diminished if more than one foregoing vernier is displayed (Figure 5). Performance for offset discrimination follows the subjective experience: With increasing number of verniers, performance deteriorates.

For example, in the five-vernier condition (threshold, 162.0") thresholds were about three times higher than in the single-vernier condition (threshold, 58.2"). Increasing the target energy seems to have increased thresholds. We attribute this effect to lateral interactions between neighboring neurons mutually weakening the activity evoked by the verniers (see Figure 1). 

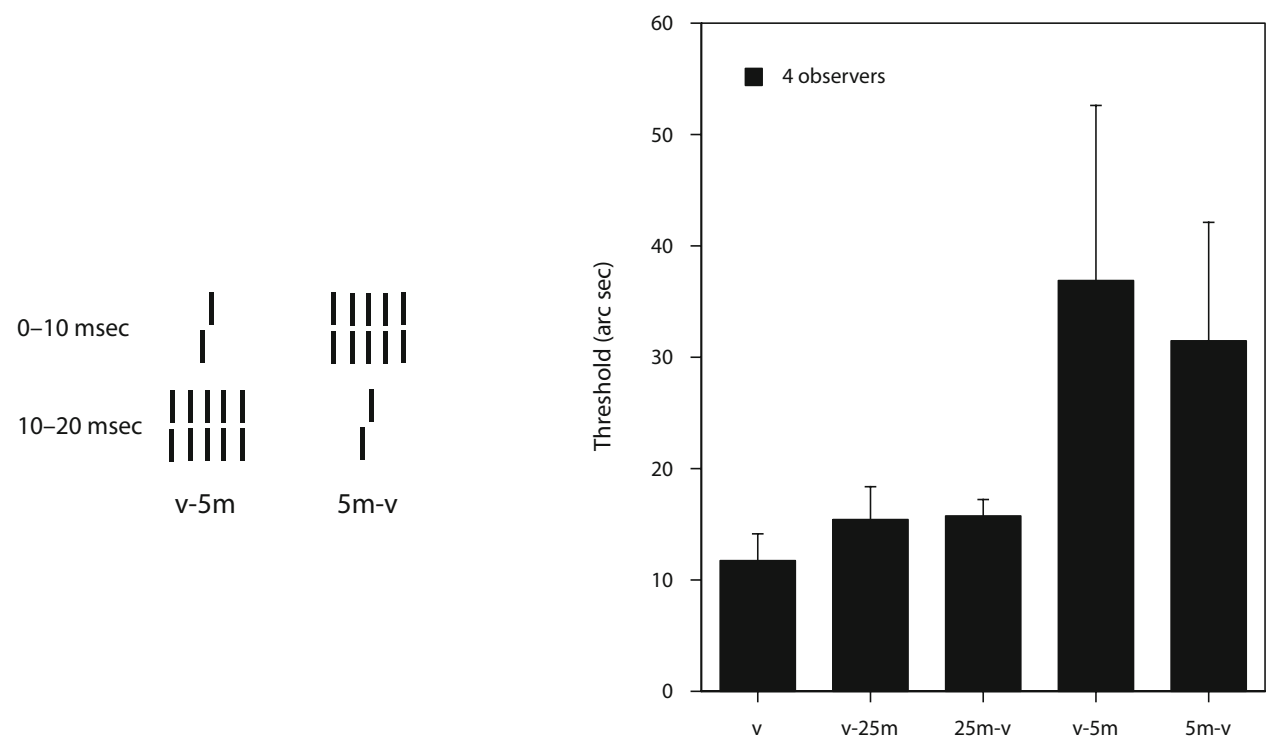

Figure 4. The target vernier was presented alone (v), preceded or followed by a masking grating of either 5 $(\mathrm{v}-5 \mathrm{~m} ; 5 \mathrm{~m}-\mathrm{v})$ or $25(\mathrm{v}-25 \mathrm{~m} ; 25 \mathrm{~m}-\mathrm{v})$ elements, respectively. Presentation times of the vernier and the grating were identical. Performance is comparable irrespective of whether the gratings precede or follow the vernier. Performance differences between the corresponding 5- and 25-element grating conditions are mainly due to 1 participant whose performance strongly deteriorated for the 5-element grating in comparison with the 25-element grating. Note the change in the scale of the ordinate relative to that for Figures 2 and 3. Observers were the same as in the previous figures.

$0-10 \mathrm{msec}$

$10-310 \mathrm{msec}$
III IIIIIIIIIIIIIIIIIIIIII

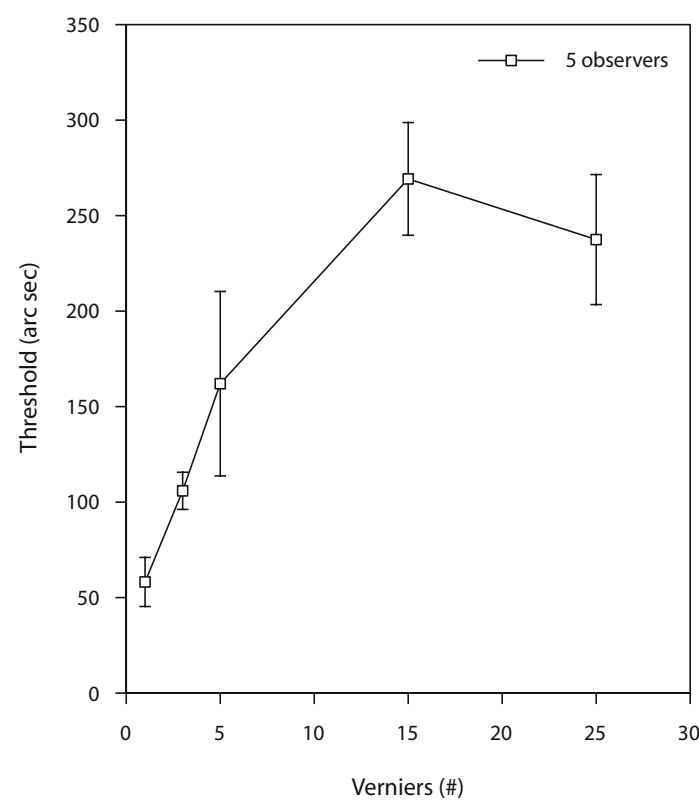

Figure 5. A varying number of offset target verniers preceded the standard grating comprising 25 elements. Thresholds increase with the number of verniers presented. Performance deteriorates in an almost monotonic fashion, asymptoting around 15 elements. Note the change in the scale of the ordinate relative to that for Figure 4. Means and standard errors are represented for 5 observers. 


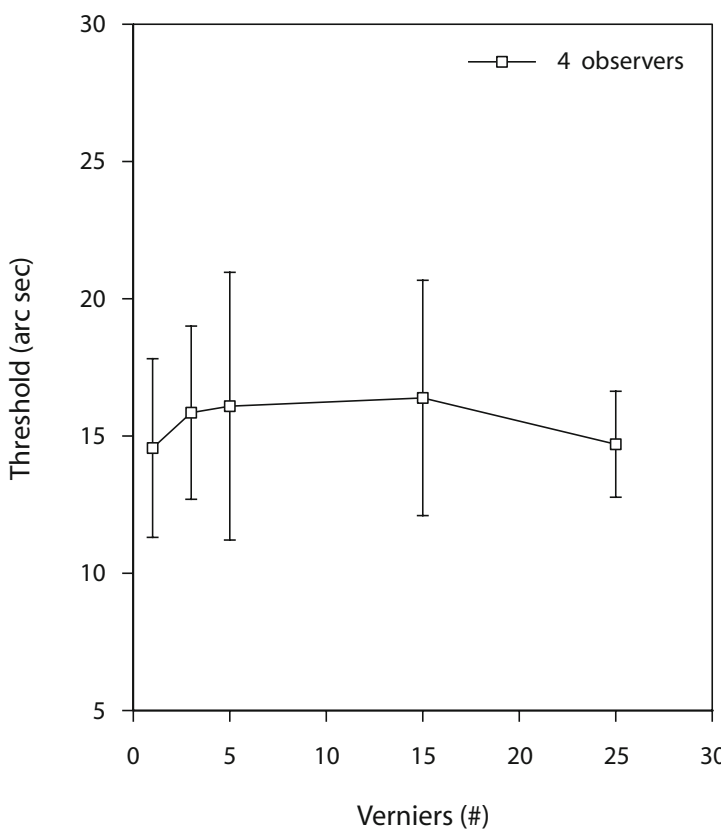

Figure 6. A varying number of verniers was presented without the standard grating. On average, the number of verniers has no strong influence on performance. Note the change in scale of the ordinate relative to that for Figure 5. Means and standard errors are presented for 4 observers.

When no grating followed, performance for vernier arrays remained almost constant irrespective of the number of verniers (Figure 6). However, intra- and interindividual performance differences were high.

\section{When Is a Grating a Grating?}

Arrays of verniers yield worse performance than does one single vernier when followed by the standard grating (Figure 5). We varied the spacing between five foregoing verniers in order to determine the transition from processing the verniers as a grouped array to processing the verniers as single entities.

\section{Method}

Five verniers, simultaneously displayed, preceded the standard grating. We changed the horizontal spacing between verniers in multiples of $200^{\prime \prime}$ (in Figure 7, a spacing of $400^{\prime \prime}$ is shown in the left panel). Therefore, verniers were always presented at positions covered by standard grating elements. Performance was also determined for a single target vernier. Four observers participated.

\section{Results and Discussion}

With increasing spacing, performance improved, reaching the performance level of the standard condition for a spacing of about 1,200" (Figure 7; cf. Westheimer \& Hauske, 1975).

\section{Grating Size}

Shine-through is not experienced when less than seven elements follow after a single target vernier. Instead, fea- ture inheritance may occur: The foregoing vernier remains invisible but bequeaths its offset to the grating that appears to be offset in the direction of the vernier (see Herzog \& Koch, 2001). Observers usually attend not to the center of the grating but focus attention on one edge element of the grating. Feature inheritance and shine-through, while differing only in the number of grating elements, represent two different stages of feature binding. In shine-through, the shine-through element is an entity independent of the grating, preserving the features of the vernier. In feature inheritance, the features of the masking grating and of the preceding vernier are bound together (Herzog \& Koch, 2001). Here, we will show that changing the binding state from shine-through to feature inheritance qualitatively changes results: More vernier targets yield better performance.

\section{Method}

We used arrays of offset verniers of 1, 3, and 5 elements. A grating with only 5, instead of 25, elements followed, lasting for $300 \mathrm{msec}$. The vernier presentation time was the minimal time for shinethrough (i.e., too short for observers to perceive a strong feature inheritance effect for one preceding vernier; see Figure 8).

\section{Results and Discussion}

Compared with performance for a grating with 25 elements, performance improved if more verniers preceded a 5-element grating (Figure 8). Subjectively, the verniers remained invisible. As is usual with feature inheritance, observers looked to one of the edge elements of the grating, where they perceived an illusory offset (Herzog \& Koch, 2001).

The increase in performance might be explained by an increase of target energy or by a reduced distance of the outermost vernier to the focus of attention (i.e., one of the edge elements; see Figure 1 and Herzog \& Koch, 2001). Hence, increasing the number of preceding verniers exerts opposite effects in shine-through and feature inheritance.

\section{GENERAL DISCUSSION}

Herzog, Fahle, and Koch $(2001,2002)$ argued that shine-through can be explained in terms of perceptual grouping. For example, a grating with two gaps is perceptually grouped into three smaller gratings (grouping by proximity; Figure 1). Shine-through does not occur, since the central of the three gratings comprises only five elements; and for small gratings, shine-through is largely abolished (Figure 1; Herzog, Fahle, \& Koch, 2001).

Grouping is a term of perceptual organization, concerning how an observer subjectively binds single elements, which are entities themselves, into a coherent object. On a neural description level, we showed with computer simulations that a first step of this grouping may indeed be realized by dynamic lateral interactions (Hermens et al., 2008; Herzog, Ernst, et al., 2003). This processing may well occur at a very early neural processing stage, such as in the primary visual cortex V1, and does not require any specialized grouping modules.

In this study, we started to investigate the underlying temporal mechanism of this tentative grouping by present- 


\begin{tabular}{|c|c|}
\hline $0-10 \mathrm{msec}$ & \\
\hline $10-310 \mathrm{msec}$ & IIIIIIIIIIIIIIIIIIIIII \\
\hline
\end{tabular}

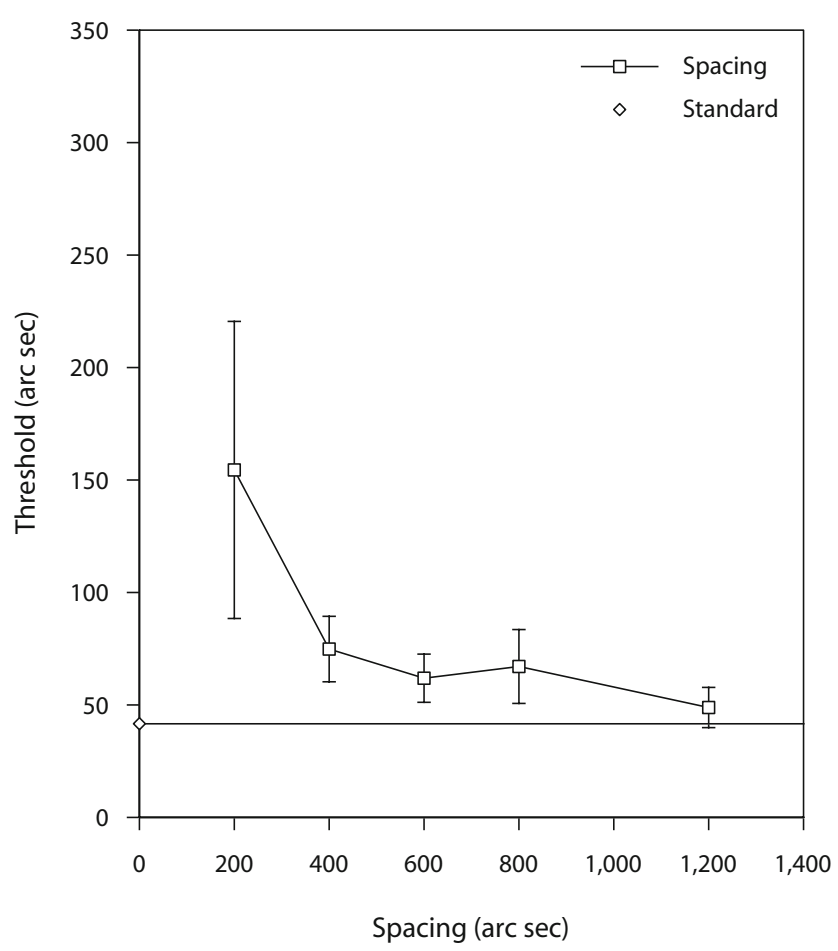

Figure 7. Five verniers preceded the standard grating. Their spacing was varied in multiples of $200^{\prime \prime}$. Performance improves with increasing spacing between verniers. The horizontal line shows performance for the standard condition with a single target vernier (the standard error is smaller than the symbol size depicted at 0 on the abscissa). Means and standard errors are presented for 4 observers.

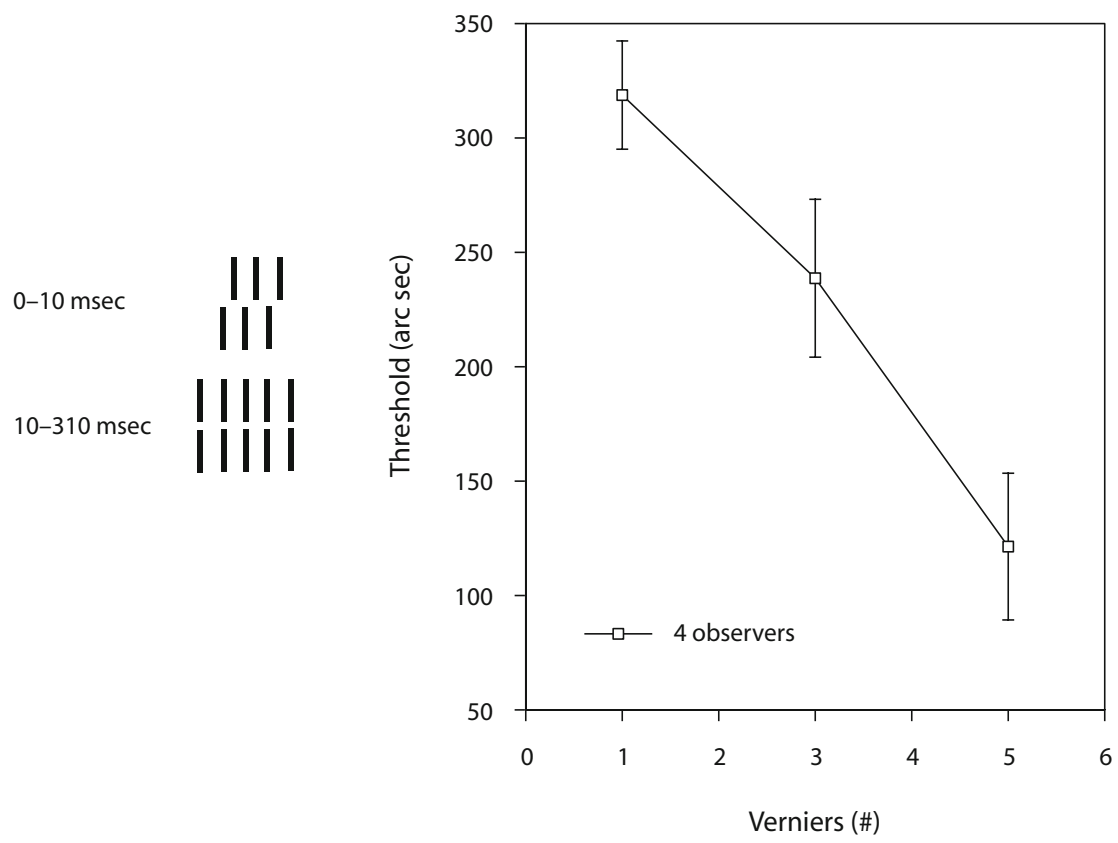

Figure 8. One, three, or five verniers preceded a grating with five elements. Performance improves if the number of verniers increases. Four observers participated. 
ing sequences of gratings. It seems that the spatial structure of a grating can be "detected" even with very brief exposures of this grating. We have shown that performance changes strongly and nonmonotonically, with the number of elements of a grating presented interleaved between the target vernier and the standard grating (Figure 2; cf. Herzog et al., 2001a). Strongest interference of vernier offset discrimination occurs for such an interleaved grating with five elements (Figure 2). Performance for interleaved gratings with more than five elements improves, possibly because of reduced interactions between the edges of the interleaved grating and the vernier. Interestingly, a three-element grating yields better results than does a five-element grating, even though the edges of the three-element grating are closer to the vernier. Computer simulations show that this result can be explained well by the spatial width of the lateral interactions (Hermens et al., 2008). Hence, the first experiment showed fast and spatially complex processing in accordance with the first hypothesis.

These interactions may also explain why arrays of target verniers yield worse performance than one single vernier even though more verniers have more target energy (a higher number of photons) than a single vernier (Figure 5). Neighboring verniers mutually weaken the neural activity of their representations, confirming the third hypothesis. We would have expected that the edges of the vernier arrays could be detected because of a higher corresponding activity. This was not the case (observers were free to base their decisions on any cue). Perhaps some neural inhibition is sufficient to prevent this effect. For longer presentation times, indeed, performance improves, and participants base their decision on the edges of the vernier arrays (results not shown), as they may do when the arrays are not masked by the standard grating (Figure 6). Our masking paradigm shows that spatial interactions are of considerable width, because separations of $1,200^{\prime \prime}$ between verniers are necessary for participants to reach a performance level comparable to that for a single vernier (Figure 7).

It might be argued that vernier arrays and gratings are processed in different "channels" than those for a single vernier, perhaps by neurons tuned to different spatial frequencies. For this reason, arrays and gratings may mask each other more strongly than a single vernier and an extended grating. However, if this is true, briefly presented gratings should interact with the vernier weakly, and, more importantly, forward and backward masking should yield similar results. However, this was not the case. Temporal order matters strongly (see Figures 2 and 3 ).

If the very same backward masking gratings are presented before the target vernier, performance surprisingly shows a floor effect independent of the number of elements, except in the single-element condition (Figure 3). This result can hardly be explained by lateral interactions quickly suppressing the activity of redundant, inner elements of gratings (second hypothesis). We would have expected that a 25 -element grating presented for $10 \mathrm{msec}$ (one or two frames of presentation) would elevate threshold only slightly, if at all, taking into ac- count that the standard grating is presented for $300 \mathrm{msec}$. However, this was not the case.

These results are surprising from other perspectives as well. First, forward masking is usually weaker than backward masking for displays with one target and one mask only (for a review, see Breitmeyer \& Öğmen, 2006). In our experiments with two masks, however, forward masking was stronger than backward masking (however, forward and backward masking yield comparable results if the standard grating is omitted; see Figure 4). Second, higher energy masks (larger size, higher contrast or luminance, etc.) yield usually worse performance than lower energy masks, contrary to our and others' findings (Herzog \& Fahle, 2002; Herzog \& Koch, 2001; Kolers, 1962; Li, Thier, \& Wehrhahn, 2000; Macknik \& Haglund, 1999; Schiller \& Greenfield, 1969; Sturr, Frumkes, \& Veneruso, 1965; Wehrhahn, Li, \& Westheimer, 1996). Our present results show that these mask size effects depend on the temporal order of presentation occurring in backward (Figure 2) but not forward (Figure 3) masking. Third, many models of object recognition simply compute the respective energies of the target and masking elements independent of their temporal order. However, this was not the case in our experiments (Figures 2 and 3).

The perceived shine-through element is not simply a fusion product of the single vernier and the central element of the standard grating, but rather an independent entity. This becomes, for example, evident in the condition in which 25 offset verniers precede the 25 -element standard grating composed of aligned verniers (Figure 5). No shine-through occurs. If we assume a fusion of verniers and grating elements on a one-by-one basis, fusion must occur for the central element of the array of 25 offset verniers comparable to that for the single vernier in the standard condition. However, this was not the case (cf. Herzog, Harms, et al., 2003; Herzog, Koch, \& Fahle, 2001b).

Masking of gratings by gratings has been studied previously (e.g., Sekuler, 1965). However, most investigations use simultaneous masking paradigms (Smith \& Swift, 1985; Swift \& Smith, 1983). Our backward masking paradigm reveals processes that are not visible in simultaneous masking (see, e.g., Figures 2 and 3 vs. 4 and 5 vs. 6). Hence, forward and backward masking paradigms allow us to investigate with great detail the temporal and spatial constraints of grouping processes that may not be visible otherwise.

\section{AUTHOR NOTE}

We thank Marc Repnow for help with the experimental setup. Correspondence concerning this article should be addressed to M. H. Herzog, Laboratory of Psychophysics, Brain Mind Institute, École Polytechnique Fédérale de Lausanne (EPFL), Station 15, CH-1015 Lausanne, Switzerland (e-mail: michael.herzog@epfl.ch).

\section{REFERENCES}

BACH, M. (1996). The "Freiburg Visual Acuity Test": Automatic measurement of visual acuity. Optometry \& Vision Science, 73, 49-53.

Breitmeyer, B. G., \& ÖĞMen, H. (2006). Visual masking: Time slices 
through conscious and unconscious vision. Oxford: Oxford University Press.

CAPUTO, G. (1996). The role of the background: Texture segregation and figure-ground segmentation. Vision Research, 36, 2815-2826.

Hermens, F., Luksys, G., Gerstner, W., Herzog, M. H., \& Ernst, U. (2008). Modeling spatial and temporal aspects of visual backward masking. Psychological Review, 115, 83-100.

Herzog, M. H., Ernst, U., Etzold, A., \& Eurich, C. (2003). Local interactions in neural networks explain global effects in gestalt processing and masking. Neural Computation, 15, 2091-2113.

Herzog, M. H., \& FAHLE, M. (2002). Effects of grouping in contextual modulation. Nature, 415, 433-436.

Herzog, M. H., Fahle, M., \& Koch, C. (2001). Spatial aspects of object formation revealed by a new illusion, shine-through. Vision Research, 41, 2325-2335.

Herzog, M. H., FAHLE, M., \& Koch, C. (2002). Spatial aspects of object formation revealed by a new illusion, shine-through [Erratum]. Vision Research, 42, 271.

Herzog, M. H., Harms, M., Ernst, U., Eurich, C., Mahmud, S., \& FAHLE, M. (2003). Extending the shine-through effect to classical masking paradigms. Vision Research, 43, 2659-2667.

Herzog, M. H., \& Косн, C. (2001). Seeing properties of an invisible object: Feature inheritance and shine-through. Proceedings of the $\mathrm{Na}$ tional Academy of Sciences, 98, 4271-4275.

Herzog, M. H., Koch, C., \& Fahle, M. (2001a). Shine-through: Temporal aspects. Vision Research, 41, 2337-2346.

Herzog, M. H., Koch, C., \& FAHLE, M. (2001b). Switching binding states. Visual Cognition, 8, 623-636.

KolERS, P. A. (1962). Intensity and contour effects in visual masking. Vision Research, 2, 277-294.

Kurylo, D. D. (1997). Time course of perceptual grouping. Perception \& Psychophysics, 59, 142-147.

Li, W., Thier, P., \& Wehrhahn, C. (2000). Contextual influence on orientation discrimination of humans and responses of neurons in V1 of alert monkeys. Journal of Neurophysiology, 83, 941-954.

MacKAY, D. M. (1973). Lateral interaction between neural channels sensitive to texture density. Nature, 245, 159-161.

MaCKNIK, S. L., \& Haglund, M. M. (1999). Optical images of visible and invisible percepts in the primary visual cortex of primates. Proceedings of the National Academy of Sciences, 96, 15208-15210.

Macknik, S. L., Martinez-Conde, S., \& Haglund, M. M. (2000). The role of spatiotemporal edges in visibility and visual masking. Proceedings of the National Academy of Sciences, 97, 7556-7560.

McCarter, A., \& Roehrs, T. (1976). A spatial frequency analogue to mach bands. Vision Research, 16, 1317-1321.

Palmer, S. E., Brooks, J. L., \& Nelson, R. (2003). When does grouping happen? Acta Psychologica, 114, 311-330.

SAGI, D., \& HochsteIN, S. (1985). Lateral inhibition between spatially adjacent spatial-frequency channels? Perception \& Psychophysics, 37, 315-322.

Schiller, P. H., \& Greenfield, A. (1969). Visual masking and the recovery phenomenon. Perception \& Psychophysics, 6, 182-184.

SEKULER, R. W. (1965). Spatial and temporal determinants of visual backward masking. Journal of Experimental Psychology, 70, 401-406.

Smith, A., \& Swift, D. J. (1985). Spatial-frequency masking and Birdsall's theorem. Journal of the Optical Society of America A, 2, 1593-1599.

Sturr, J. F., Frumkes, T. E., \& Veneruso, D. M. (1965). Spatial determinants of visual masking: Effect of mask size and retinal position. Psychonomic Science, 3, 327-328.

SwiFT, D. J., \& Sмiтh, A. (1983). Spatial frequency masking and Weber's law. Vision Research, 23, 495-505.

TAYlor, M. M., \& Creelman, C. D. (1967). PEST: Efficient estimates on probability functions. Journal of the Acoustical Society of America, 41, 782-787.

Wehrhahn, C., Li, W., \& Westheimer, G. (1996). Patterns that impair discrimination of orientation in human vision. Perception, 25, 1053-1064.

Westheimer, G., \& Hauske, G. (1975). Temporal and spatial interference with vernier acuity. Vision Research, 15, 1137-1141.

ZHAOPING, L. (2003). V1 mechanisms and some figure-ground and border effects. Journal of Physiology, 97, 503-515.

(Manuscript received February 6, 2004; revision accepted for publication February 11, 2008.) 